\title{
Nerve growth factor promotes reparative angiogenesis and inhibits endothelial apoptosis in cutaneous wounds of Type 1 diabetic mice
}

\author{
G. Graiani $^{1}$ C. Emanueli ${ }^{2}$ E. Desortes ${ }^{2}$ S. Van Linthout ${ }^{2}$ A. Pinna ${ }^{2}$ C. D. Figueroa ${ }^{3}$ L. Manni ${ }^{4}$ P. Madeddu M. $^{2}$ \\ ${ }^{1}$ Department of Pathology, University of Parma, Italy \\ ${ }^{2}$ Experimental Medicine and Gene Therapy Section, National Institute of Biostructures and Biosystems (INBB), 07033, \\ Osilo (Sassari), Italy \\ ${ }^{3}$ Department of Pathology, Austral Universidad, Valdivia, Chile \\ ${ }^{4}$ Institute for Cardiovascular Research, Wallenberg Laboratory, Sahlgrenska University Hospital/Sahlgrenska, Göteborg, Sweden \\ ${ }^{5}$ Internal Medicine, University of Sassari, Italy
}

\section{Abstract}

Aims/hypothesis. The neurotrophin nerve growth factor (NGF) is pro-angiogenic and facilitates wound repair. The present study was conducted to (i) assess the statement of NGF system components in diabetic wounds and (ii) evaluate whether NGF supplementation could prevent impairment of wound neoangiogenesis by diabetes.

Methods. Skin wounds were produced in the interscapular region of streptozotocin-induced diabetic mice. NGF ( $1 \mu \mathrm{g}$ per day in PBS) or vehicle was applied onto the ulcers for 3 days after punching. Nondiabetic mice were used as controls.

Results. In wounds of untreated diabetic mice, endogenous levels of immunoreactive NGF were lower than those in wounds of non-diabetic mice $(p<0.01)$. Immunohistochemical analysis showed down-regulation of tyrosine kinase receptor-A (TrkA) and up-regulation of p75 receptor in granulation tissue microvasculature. Local NFG administration prevented diabetesinduced expressional alterations, enhanced reparative capillarisation $(p<0.01)$, and accelerated wound closure $(p<0.01)$. This was associated with a three-fold increase in endothelial cell proliferation $(p<0.01)$, while apoptosis was reduced by $50 \%(p<0.05)$. Quantitative RT-PCR documented a 5.5-fold increase in the expression of vascular endothelial growth factor-A (VEGF-A) by exogenous NGF in diabetic tissues $(p<0.01)$. In in vitro preparations of human endothelial cells from derma, NGF increased the release of immunoreactive VEGF-A, and reduced high-glucose-induced apoptosis $(p<0.05)$, the latter effect being inhibited by a VEGF-A receptor-2 antagonist.

Conclusions/interpretation. Diabetic ulcers display distinct alterations in reparative angiogenesis and in the expression of NGF and its receptors. NGF supplementation corrects endogenous liabilities, facilitates vascular regeneration, and suppresses endothelial apoptosis seemingly via VEGF-A. Our findings unravel new mechanisms responsible for NGF reparative action.

Keywords Angiogenesis - Apoptosis - Diabetes · Vascular growth factor $\cdot$ Wound healing
Received: 31 December 2003 / Accepted: 27 February 2004

Published online: 26 May 2004

(C) Springer-Verlag 2004

P. Madeddu ( $)$

Experimental Medicine and Gene Therapy Section,

National Institute of Biostructures and Biosystems (INBB),

Viale S. Antonio, 07033, Osilo (Sassari), Italy

E-mail: madeddu@yahoo.com

Tel.: +39-079-3441006, Fax: +39-079-3441006

Abbreviations: EC, endothelial cell - NGF, nerve growth factor TUNEL, terminal-deoxynucleotidyl-mediated deoxyuridine5'-triphosphate nick-end labelling · TrkA, tyrosine kinase receptor-A · VEGF-A, vascular endothelial growth factor-A

\section{Introduction}

Foot ulcers represent a serious clinical problem for the diabetic patient. Diabetic wounds repair very slowly, cause physical inability, and often require amputation. These problems are ultimately caused by poor metabolic control, over time leading to microvascular damage, nerve damage and reduced resistance to infection [1]. Up to the present, healing treatments of diabetic ulcers have not delivered satisfactory results. Dissecting the mechanisms responsible for the delayed repair may offer new potential targets for treatment. For instance, evidence is accumulating that in diabetes, an 
insufficient surge of endothelial growth factors $[2,3$, 4] and primary alterations in endothelial cell (EC) biology, namely activation of apoptotic death $[2,5,6]$, may hinder reparative neovascularisation, a host defence response necessary to restore oxygen and nutrient support to the healing tissue.

Nerve growth factor (NGF) represents the first isolated and best-characterised member of a growing family of neurotrophins which includes brain-derived neurotrophic factor and neurotrophins $3-5$ [7]. NGF is known to regulate the survival and differentiation of neurons. In animal models of diabetes, NGF is depleted in peripheral nerves and this deficit has been related to degeneration or impaired regeneration of peripheral nerves, and to dysfunction of sensory small-diameter fibres that release substance P [8]. Supplementation with exogenous NGF reverses the pathological changes typical of diabetic neuropathy [9]. In addition to its effects on nerve cells, NGF has a potent healing effect on otherwise untreatable corneal wounds and pressure ulcers, seemingly through stimulation of reepithelialisation and granulation tissue formation $[10$, $11,12,13]$.

Recent findings suggest that NGF curative properties might be related to previously unsuspected involvement of the neurotrophin in the regulation of vascular growth. We showed that NGF is pro-angiogenic and promotes tissue healing in a mouse model of limb ischaemia, thus providing the first demonstration of a neural drive for reparative angiogenesis [14]. NGF has direct control of vascular biology through its high-affinity tyrosine kinase receptor-A (TrkA) and low-affinity p75 receptor expressed on ECs [15] and vascular smooth muscle cells [16]. The relative abundance of NGF subtype receptors might provide a bifunctional switch for life or death situations [17]. In fact, TrkA phosphorylation starts a cascade of events that via activation ERK1/2 and vascular endothelial growth factor-A (VEGF-A)/phosphatidyl-3' kinase/ Akt-B/endothelial nitric oxide synthase pathways, leads to stimulation of cell proliferation $[14,18]$. Conversely, p75 activation induces apoptosis, especially under conditions where TrkA activation is reduced or absent $[19,20]$. Besides being a biological target for neurotrophins, vascular ECs actively produce NGF $[14,15]$, thus suggesting the existence of paracrine/autocrine loops of NGF on these cells.

Based on the above rationale, we designed a study to investigate whether the expression of endogenous NGF system is deregulated in skin ulcers of Type 1 diabetic mice. In addition, we examined whether NGF supplementation can prevent the cellular and molecular liabilities that hamper proper reparative angiogenesis in Type 1 diabetes.

\section{Materials and methods}

Diabetes induction and operative model. All procedures were carried out in accordance with the Guide for the Care and Use of Laboratory Animals (1996; Institute of Laboratory Animal Resources, National Academy of Sciences, Bethesda, Md., USA).

Male CD1 mice (Charles River, Comerio, Italy) were made diabetic using streptozotocin (40 mg/kg i.p. per day for 5 days; Sigma, Milan, Italy). Only mice showing consistently elevated fasting glucose levels $(13.8 \mathrm{mmol} / \mathrm{l})$ and overt glycosuria were included in the study. At 4 weeks from induction of diabetes, mice (aged 12 weeks) were anaesthetised with 2,2,2-tribromoethanol ( $880 \mathrm{mmol} / \mathrm{kg}$ body weight i.p.; Sigma) to receive two full-thickness 4-mm-wide skin wounds in the interscapular region by means of disposable skin punch equipment. Agematched, non-diabetic mice were used as controls.

NGF supplementation protocol. NGF was prepared from adult male mouse submandibulary glands [21] and further purified by additional carboxymethyl-cellulose chromatography. The purified NGF was dissolved in PBS and kept at $4{ }^{\circ} \mathrm{C}$ for no longer than 1 week. Under these conditions, both stability and activity of NGF remains unchanged.

Starting immediately after punching and continuing for 3 days, NGF ( $1 \mu \mathrm{g}$ in $20 \mu \mathrm{l}$ PBS) was daily applied to the rightside ulcer, while an equal volume of PBS was added to the contralateral wound ( $n=19$ mice). We adopted a previously published short-term treatment schedule [13] because our aim was to evaluate neoangiogenesis, a process that occurs at early phases of healing. The dosage of NGF corresponds with that previously employed in diabetic patients with trophic ulcers [11]. In addition, non-diabetic controls $(n=10)$ were subjected to the same wounding procedure and then randomised to treatments described above.

Clinical outcome was established by determining the rate of wound closure. To do this, wound area was measured immediately after punching, and then sequentially until the mice were killed, by means of a morphometry program (ProPlus Image Software) on images captured by a video camera (Pixera), or using a caliper to determine two perpendicular diameters of the wound. In our experience, the two methods work very well together in estimating wound surface and the rate of closure.

Histological assessment of microvascular effects. The impact of diabetes and exogenous NGF supplementation on reparative capillarisation was examined in wounded tissue. These experiments were also aimed at determining the concurrent expressional changes of endogenous NGF system. For this purpose, the mice were killed with an overdose of anaesthetic. Wounds and surrounding skin were removed and perpendicularly cut into two halves. One half was immediately frozen for molecular biology studies, which were conducted on specimens collected at 3 days from punching (vide infra), and the other was fixed in $4 \%$ buffered formalin solution and further processed for histology and/or immunohistochemical analysis on samples harvested at 3 and 14 days. A piece of non-wounded skin was taken for reference. Analysis was performed by a scientist (G. Graiani) who was unaware of the randomisation protocol.

Sections were cut and stained with hematoxylin and eosin. In addition, a second set of sections was stained with antibody to platelet endothelial cell adhesion molecule (PECAM [CD31]; Dako, Glostrup, Denmark). Immunostaining for PECAM was revealed with streptavidin-conjugated peroxidase (SCP) and $\mathrm{DAB}$ as a chromogen. Capillaries and cellular components were counted using an ocular reticle $\left(9604-\mu \mathrm{m}^{2}\right.$ area) at $\times 1000$ magnification. For each section, more than 50 fields 
were randomly examined and averaged. The number of capillaries per field was used to compute capillary density per $\mathrm{mm}^{2}$ of section.

Analysis of apoptosis. Apoptosis was assessed by terminal deoxynucleotidyl transferase-mediated dUTP nick-end labelling (TUNEL) assay in wounded tissues of diabetic or non-diabetic mice ( $n=6$ per group). Sections $3 \mu \mathrm{m}$ thick were stained with SCP/DAB and counterstained with hematoxylin. Positive controls were pre-treated with $1 \mu \mathrm{g} / \mathrm{ml}$ DNase I, while negative controls were incubated in the absence of terminal deoxynucleotidyl-transferase. Sections were examined at $\times 1000$ magnification. TUNEL-positive ECs were counted and expressed as number per $\mathrm{mm}^{2}$ of section or per 1000 capillaries.

The NGF $\mathrm{p} 75$ receptor has been linked to apoptosis. Therefore, we determined the rate of apoptotic ECs among those positive or negative for $\mathrm{p} 75$. To do this, sections were incubated with $5 \mathrm{U}$ of $2.5 \% \mathrm{CoCl}_{2}, 0.2 \mathrm{~mol} / \mathrm{l}$ potassium cacodylate, $25 \mathrm{~mol} / \mathrm{l} \mathrm{Tris}-\mathrm{HCl}, 0.25 \% \mathrm{BSA}$, and $0.5 \mathrm{nmol} / \mathrm{l}$ biotinylated $2^{\prime}$ deoxyuridine-5'-triphosphate (biotin-16-dUTP). Apoptotic nuclei were revealed by green fluorescence after incubation with $5 \mu \mathrm{g} / \mathrm{ml}$ of FITC-labelled extravidin (Sigma). Subsequently, sections were exposed to anti-p75 antibody (mouse mab, 1:50; Dako) for $1 \mathrm{~h}$ at $37^{\circ} \mathrm{C}$, and the reaction was visualised by the red fluorescence of TRITC-conjugated anti-mouse IgG (Sigma). Nuclei were visualised by bisbenzimide staining. The proportion of p75-positive or p75-negative ECs with apoptotic nuclei was then calculated under fluorescence microscopy. Since the TUNEL technique may alter tissue preservation, only nuclei lining the vessel luminal profiles were considered to belong to ECs.

BrdU labelling for measurement of EC proliferation. An additional experiment was performed to determine whether NGF stimulates EC proliferation in vivo. Proliferation was assessed by immunohistochemical analysis of the cellular incorporation of thymidine analogue BrdU. Diabetic mice were randomly allocated to wound treatment with NGF $(n=5)$ or PBS $(n=4)$ and at the same time received $\mathrm{BrdU}(25 \mathrm{mg} / \mathrm{kg}$ body weight i.p. in $100 \mu \mathrm{l}$ of PBS per day for 3 days; Sigma). Three days after punching, mice were killed and the wounded skin harvested for analysis. Proliferating cells were identified with a monoclonal mouse antibody to BrdU (1:100; Dako) diluted in PBS + $1 \%$ normal goat serum. Unspecific background staining was eliminated by pre-incubation for $20 \mathrm{~min}$ in 3\% peroxidase and $20 \mathrm{~min}$ in methanol. Sections were then incubated with secondary anti-mouse antibody (Dako), stained with SCP/DAB, and counterstained with hematoxylin. For each section, more than 50 fields were randomly examined and averaged.

Effect of diabetes on NGF and NGF receptor expression. TrkA and p75 receptor expression was evaluated in specimens harvested from diabetic or non-diabetic mice at 3 days from punching ( $n=6$ per group). Identification of TrkA receptor was performed using a polyclonal rabbit antibody (Santa Cruz). The specific binding was detected using a biotinylated antirabbit IgG and the streptavidin-peroxidase technique (Vectastain Elite ABC kit, Vector Laboratories). Similarly, low affinity p75 receptor was identified using a monoclonal mouse antibody (1:50; Dako) diluted in PBS + 1\% normal goat serum. In negative controls, the primary antibody was omitted. For each section, more than 50 fields were randomly examined and averaged. The number of TrkA or p75-positive cells per field was counted to compute density per $\mathrm{mm}^{2}$ section. To assess the relative abundance of NGF receptor expression in wound microvasculature, the proportion of TrkA- or p75-positive ECs over 1000 capillaries was calculated.
NGF content was determined in fresh tissue homogenates using a highly sensitive ELISA (NGF $\mathrm{E}_{\max }$ ImmunoAssay System, Promega).

Analysis of VEGF-A expression by real-time quantitative $P C R$. Next, we determined the impact of diabetes and NGF treatment on the expression of the master angiogenic factor VEGF-A. Frozen skin specimens (obtained at 3 days from punching) were homogenised in Tri reagent (Sigma), and RNA was extracted according to the manufacturer's protocol. Then, $2 \mu \mathrm{g}$ of RNA in $50 \mu \mathrm{l}$ of reaction solution was reversetranscribed with M-MLV reverse transcriptase (Invitrogen). We used $5 \mu \mathrm{l}$ of transcript product solution for RT-PCR amplification. Primers for amplification of murine $V E G F-A$ cDNA (Genebank M95200) were: 5' CCAGCGAAGCTACTGCCGTCCA 3' (forward) and 5' ACAGCGCATCAGCGGCACAC $3^{\prime}$ (reverse) from which a 109-bp amplification product was obtained. VEGF-A cDNA was measured using quantitative real-time PCR (ABI PRISM 7000 Sequence Detection System) and the levels of VEGF-A cDNA were normalised to the levels of the glyceraldehyde-3-phosphate-dehydrogenase housekeeping gene cDNA. As DNA standards, the products of the conventional PCR for murine VEGF (111 bp) and GAPDH (156 bp) with the use of the primers designed for the real-time PCR, were cloned into pGEM-T Easy vector (Promega).

In vitro proliferation and apoptosis assays on ECs from human derma microvasculature. The effects of NGF on EC biology were evaluated under culture conditions simulating diabetic hyperglycaemia. Human ECs were kindly provided by G. Alessandri (Besta Institute, Milan, Italy). Proliferation experiments were performed by the MTS assay (Promega). Human ECs (at 8th passage) were seeded in EGM-2 EC Medium-2 (Cambrex) on 96-well plates pre-coated with collagen and fibronectin. After $4 \mathrm{~h}$, medium was exchanged for a similar one that contained reduced FBS and high glucose $(30 \mathrm{mmol} / \mathrm{l})$ plus NGF $(100 \mathrm{ng} / \mathrm{ml})$ or vehicle. Proliferation was assessed after 24 hours. Each experiment was performed in six wells per experimental group and repeated three times.

Finally, the effect of NGF on high-glucose-induced apoptosis was examined. Human ECs were plated onto 24-well plates and then incubated for $72 \mathrm{~h}$ in reduced FBS and high-glucose (30 mmol/l) medium containing vehicle, NGF $(100 \mathrm{ng} / \mathrm{ml})$, or NGF plus PTK787 $\left(10^{-6} \mathrm{mmol} / \mathrm{l}\right)$, a specific VEGF-A receptor 2 antagonist (Novartis Pharma). At the end of incubation, cells were fixed in $4 \%$ paraformaldehyde for $10 \mathrm{~min}$, washed twice with PBS and incubated with 4'-6'-diaminidino phenylindole (DAPI, $1 \mu \mathrm{g} / \mathrm{ml}$ PBS; Roche), a fluorescent probe for DNA, for 20 min. Wells were examined by fluorescent microscopy. Five randomly selected fields per well were captured with a video camera at $10 \times$ magnification. Apoptotic ECs, identified by condensation and fragmentation of their nuclei, were counted automatically (Image ProPlus). The total cell number was also computed in each field to calculate the percentage of apoptotic cells. Each experiment was performed in quadruplicate and repeated three times.

In separate experiments, immunoreactive VEGF-A levels in the NGF-conditioned culture media were measured using a quantitative ELISA kit (R\&D Systems).

Statistical analysis. All results are expressed as means \pm SEM. Nested ANOVA was performed to test for interaction between time and grouping factor. Differences within and between groups were determined using paired or unpaired Student's $t$ test respectively. A $p$ value of less than 0.05 was considered statistically significant. 


\section{Results}

Effects of Type 1 diabetes on the expression of endogenous NGF system and on EC proliferation and apoptosis. As shown in Figure 1a, in wounds of untreated diabetic mice, endogenous NGF levels were lower than those in wounds of non-diabetic controls $(p<0.01)$. In wound microvasculature of diabetic mice, immunohistochemical analysis documented a 13-fold reduction of TrkA-positive ECs (Fig. 1b) and a fourfold increase of p75-positive ECs (Fig. 1c). As a consequence of these reciprocal changes, TrkA : p75 ratio was strikingly reduced in diabetic mice $(0.011 \pm 0.002$ vs $1.05 \pm 0.11$ in non-diabetic mice, $p<0.001$ ).

As shown in Figure 1d, apoptosis was markedly activated in diabetic mice ( $28 \pm 5$ vs $14 \pm 2$ apoptotic ECs per 1000 capillaries in non-diabetic mice, $p<0.01)$. By immunofluorescence microscopy, we also documented that $93 \%$ of apoptotic ECs were p75-positive. In addition, diabetic wounds displayed depressed EC proliferation. In fact, during the first 3 days after punching, BrdU incorporation by ECs was significantly lower $(p<0.01)$ than in non-diabetic wounds $(7.7 \pm 1.5$ and $18.4 \pm 2.2$ BrdU-positive ECs respectively). These results indicate that Type 1 diabetes alters the expression of endogenous NGF system and impairs EC viability and proliferation in wound neovascularisation.

\section{a}
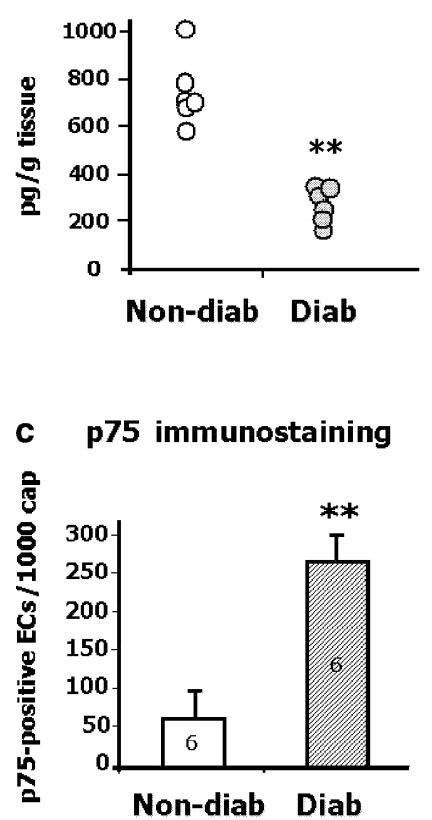
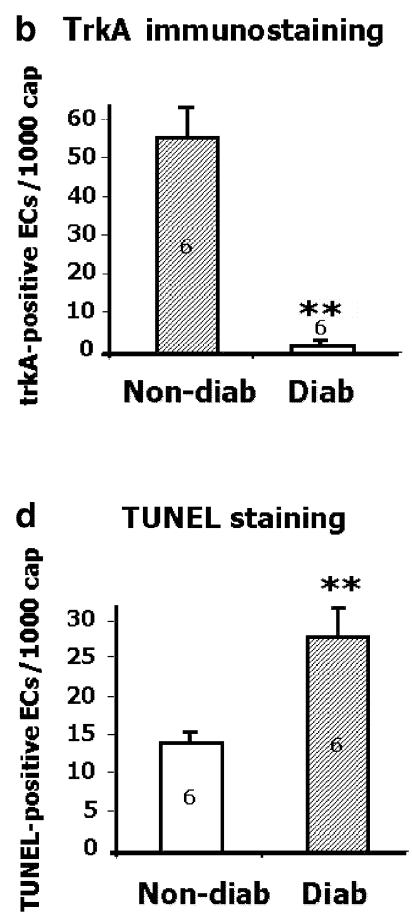

Fig. 1. Graphs show the effect of Type 1 diabetes mellitus (Diab) on NGF levels (a), and number of ECs positive for TrkA (b), p75 receptor (c), or TUNEL staining (d) in tissue sections from skin wounds. Non-diabetic mice (Non-diab) were used as controls. Specimens were collected 3 days after punching. Values are means \pm SEM. Numbers within columns indicate sample size. cap, capillaries; $* * p<0.01$ vs control
NGF accelerates the rate of wound closure. In diabetic mice, wound closure was slower than in non-diabetic mice (Fig. 2, $p<0.05$ ), but supplementation of exogenous NFG corrected the impairment (Fig. 2b). In fact, at day 2 from punching, the areas of NGFtreated diabetic wounds averaged 1.6 times less than those of contralateral PBS-treated wounds $(p<0.01)$. As a consequence of this early improvement, the rate of wound closure over the remaining period was shifted to match the pattern seen in non-diabetic controls.

NGF potentiates reparative angiogenesis. Next, we tested the effect of NGF on reparative neoangiogenesis. Capillary density of non-wounded skin did not differ between diabetic and non-diabetic mice $\left(180 \pm 49\right.$ vs $204 \pm 13$ capillaries $/ \mathrm{mm}^{2}$, NS). However, as shown in Figure 3a, wound capillarisation was significantly impaired in PBS-treated diabetic mice $\left(232 \pm 16\right.$ capillaries $\left./ \mathrm{mm}^{2}\right)$ as compared with the non-
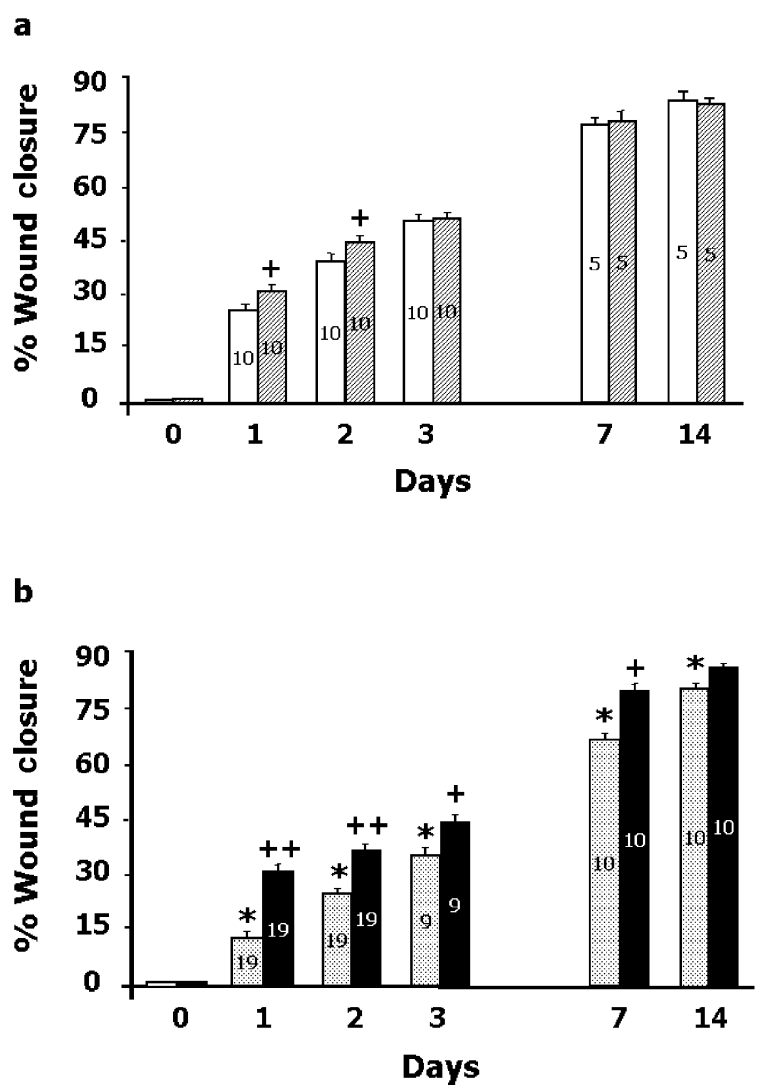

Fig. 2. Bar graph shows the rate of wound closure in non-diabetic (a) or Type 1 diabetic (b) mice. With mice under 2,2,2tribromoethanol anaesthesia, two full-thickness 4-mm-wide skin wounds were produced in the interscapular region using disposable skin punch equipment. Left-side wounds were treated with PBS (non-diabetic, empty columns; diabetic, dotted columns) and right-side wounds with NGF ( $1 \mu \mathrm{g}$ in $20 \mu \mathrm{l} \mathrm{PBS})$ (non-diabetic, hatched columns; diabetic, full columns). Values are means \pm SEM. Numbers within columns indicate sample size. ${ }^{*} p<0.05$ vs non-diabetic, ${ }^{+} p<0.05$ and ${ }^{++} p<0.01$ vs contralateral side 


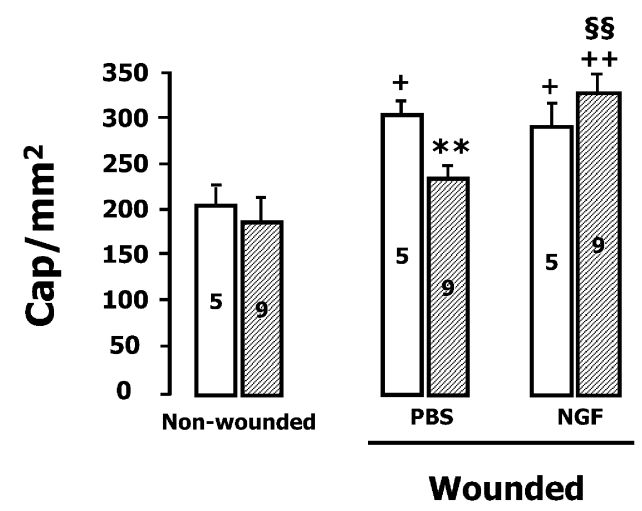

Fig. 3. Bar graph showing cutaneous capillary density of diabetic (hatched columns) or non-diabetic (empty columns) mice. Wounds were treated with PBS or NGF for 3 consecutive days. Capillary density in wounded and non-wounded skin was determined. Values are means \pm SEM. Numbers within columns indicate sample size. cap, capillaries; ${ }^{+} p<0.05$ and ${ }^{++} p<0.01$ vs non-wounded, $* * p<0.01$ vs non-diabetic, $\$ \S p<0.01$ vs PBS

\section{BrdU staining}

a

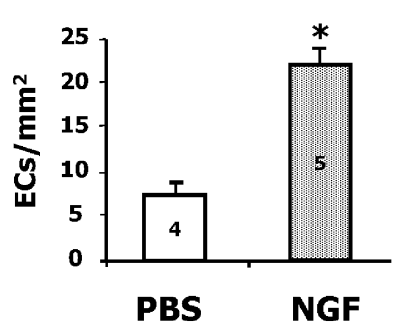

TUNEL staining

b

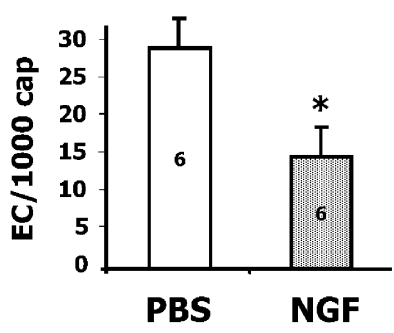

Fig. 4. Bar graph shows the number of dividing ECs, identified by BrdU staining (a), and the proportion of apoptotic ECs, identified by TUNEL staining (b), in wounded tissue harvested from diabetic mice at day 3 from punching. Wounds were treated with PBS (empty columns) or NGF (dotted columns) for 3 consecutive days. Values are means \pm SEM. Numbers within columns indicate sample size. cap, capillaries; ${ }^{*} p<0.05$ vs PBS

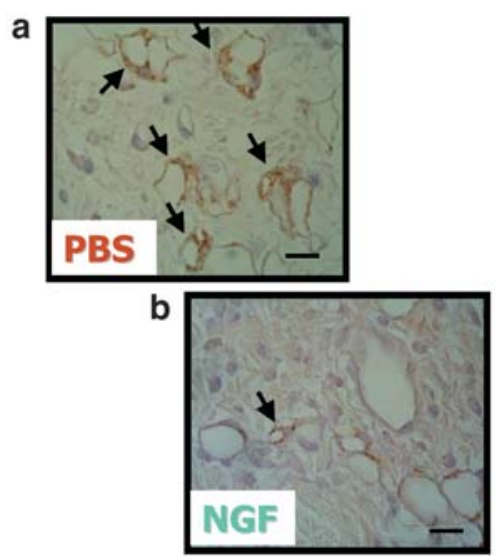

Fig. 5. Representative immunohistochemical staining $(\mathbf{a}, \mathbf{b})$ for p75 receptor in specimens of wounded tissue harvested from diabetic mice on day 3 from punching. Wounds were treated with PBS (a) or NGF (b) for 3 consecutive days. The number of p75-positive microvessels (arrows) is reduced in NGF-treat- diabetic mice $\left(291 \pm 21\right.$ capillaries $\left./ \mathrm{mm}^{2}, p<0.001\right)$. The defect in reparative angiogenesis was corrected by topical application of exogenous NGF leading the capillarity of diabetic wounds to $312 \pm 32$ capillaries $/ \mathrm{mm}^{2} \quad(p<0.01$ vs contralateral PBS-treated wounds). In contrast, NGF supplementation did not further enhance reparative neovascularisation of nondiabetic wounds (NS). These results indicate that NGF selectively stimulates reparative angiogenesis in diabetic wounds.

$N G F$ stimulates EC proliferation and suppresses apoptosis. As shown in Figure 4a, the number of BrdUpositive ECs was three-fold higher in wounds that received NGF supplementation $(21.8 \pm 3.2$ vs $7.7 \pm 1.5$ ECs per $\mathrm{mm}^{2}$ in PBS-treated wounds, $p<0.01$ ). Likewise, epithelial cell proliferation was significantly enhanced in NGF-treated wounds $(p<0.01$ vs PBS, data not shown).

As shown in Figure $4 \mathrm{~b}$, the number of apoptotic ECs was significantly reduced after 3-day treatment with NGF (14 \pm 4 vs $28 \pm 5$ apoptotic ECs per 1000 capillaries in PBS-treated wounds, $p<0.05$ ).

Therefore, exogenous NGF is able to correct the diabetes-induced deficit in EC proliferation, and protects ECs from apoptosis.

Expression of NGF receptors after exogenous NGF administration. By immunohistochemistry we examined whether NGF treatment alters the expression of its own receptors. NGF increased the number of TrkA-positive ECs in diabetic wounds (13.87 \pm 6.46 vs $1.66 \pm 1.15$ per 1000 capillaries in PBS-treated wounds, $p<0.05$ ) and reduced the number of p75-positive ECs (Fig. 5, $p<0.01$ vs PBS-treated wounds). As a consequence of these reciprocal changes, TrkA : p75 ratio was increased 19-fold in NGF-treated diabetic mice.

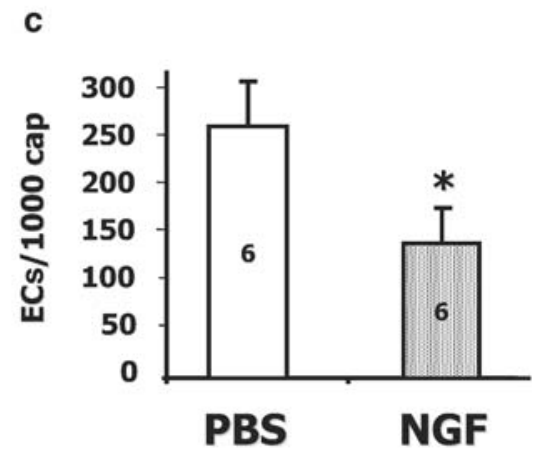

ed wounds. Signal intensity is also reduced. Original magnification: $\times 1000$. The bar length corresponds to $10 \mu \mathrm{m}$. The proportion of p75-positive ECs per 1000 capillaries is shown in $\mathbf{c}$. Numbers within columns indicate sample size. cap, capillaries; $* p<0.05$ vs PBS 


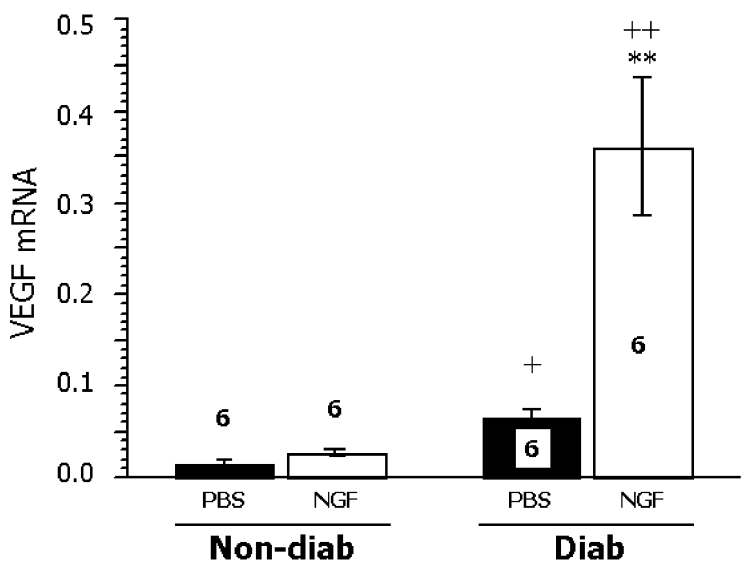

Fig. 6. Bar graph illustrates $V E G F-A$ expressional changes in wounded tissues harvested 3 days after punching from non-diabetic (Non-diab) or diabetic (Diab) mice treated with PBS (closed columns) or NGF (open columns). Numbers within columns indicate sample size. ${ }^{* *} p<0.01$ vs PBS, ${ }^{+} p<0.05$ and ${ }^{++} p<0.01$ vs non-diabetics

NGF stimulates VEGF-A expression in diabetic wounds. As shown in Figure 6, quantitative RT-PCR analysis documented a 5.5-fold increase of murine VEGF-A expression in NGF-treated wounds compared with PBS-treated wounds $(p<0.01)$. The effect of NGF on $V E G F-A$ expression was less pronounced in non-diabetic mice $(p=0.07, \mathrm{NS})$.

$N G F$ stimulates VEGF-A release in vitro and inhibits high-glucose-induced EC apoptosis. NGF supplementation increased the levels of immunoreactive VEGF in the conditioned media $(2.1 \pm 0.2$ vs $0.5 \pm 0.1 \mathrm{pg} / \mathrm{ml}$ in controls, $p<0.01$ ).

The high-glucose environment triggered apoptosis of cultured human derma ECs. Total cell counting averaged $2 \pm 1$ per field in high-glucose-containing wells, compared with $19 \pm 2$ per field in normal-glucose-containing wells $(p<0.01)$. NGF supplementation partially prevented the reduction in EC counting $(9 \pm 1$ cells per field, $p<0.01$ vs vehicle) and inhibited apoptosis induced by $72-\mathrm{h}$ incubation in high glucose ( 45 vs $86 \%$ apoptotic ECs in vehicle, $p<0.05$ ), the latter effect being reversed by an antagonist for VEGF receptor-2 (76\% apoptotic ECs, NS vs vehicle).

Under high-glucose conditions, proliferation of derma ECs was not significantly enhanced by NGF (NS).

\section{Discussion}

NGF is essential for the maturation and differentiation of the central nervous system of the embryo. It then continues to have neurotrophic and pleiotropic healing effects during adulthood [9, 10, 11, 12, 13, 14, 22]. In fact, NGF expression is up-regulated after cutaneous wounding [13] or induction of ischaemia in otherwise healthy animals [14], the functional relevance of this phenomenon being supported by the recent discovery that post-ischaemic repair is hampered by administration of anti-NGF antibodies [14].

It is worth noting that NGF production is instead impaired in the skin of rodents (as shown in the present study) and humans with Type 1 diabetes [8], suggesting that insufficient surge of the neurotrophin may be implicated in delayed repair of diabetic ulcers. The present study confirms that Type 1 diabetes causes relevant alterations in ECs within wound microvasculature, consisting of depressed proliferation and increased apoptosis [23]. Most importantly, it adds the novel information that disturbed EC biology is associated not only with low NGF content, but also with distinct expressional alterations of vascular NGF receptors resulting in a 100fold decrease of TrkA : p75 ratio. These alterations might have profound impact on EC proliferation and viability. In fact, studies in neuronal cells suggest that the mechanism that allows cell survival upon induction of mitosis is dependent on the continuous presence of NGF and the expression of TrkA receptors [24]. Binding of NGF to TrkA causes tyrosine kinase domain auto-phosphorylation, allowing interaction with adaptor proteins that couple the receptor to intracellular signalling cascades, namely the protein kinase ERK pathway, the PI3K/Akt pathway, and the phospholipase C pathway [18]. These signalling cascades trigger cell cycle progression through Shc protein recruitment and activation of Ras and Rap1 [25].

The role of p75 receptor in the control of cell cycle has not been fully determined. Activation of p75, a member of the TNF- $\alpha$ receptor superfamily, reportedly increases the affinity of NGF binding to TrkA, thereby enhancing NGF-mediated neuronal survival [24]. However, under conditions where TrkA expression is reduced (as observed in our diabetic mice), the p75 receptor preferentially triggers apoptosis $[17,19]$. Similar to the TNF- $\alpha$ receptors, the p75 has an intracellular domain, a so-called death domain, that forms complexes with zinc-finger-containing proteins, such as NRIFs, SC-1, and NRAGE, a member of the melanoma antigen family [26, 27]. Translocation of these complexes to the nucleus leads to apoptosis or cell cycle arrest via ceramide [28] and stress kinase c-jun N-terminal kinase (JNK) [27].

From the above, we speculate that shortage of NGF combined with disruption of the balance between prosurvival TrkA and pro-apoptotic p75 receptor may contribute to the insufficient neoangiogenesis of diabetic wounds. The link between p75 and EC death is further suggested by our observation that more than $90 \%$ of apoptotic ECs in granulation tissue of diabetic wounds were p75-positive. Thus, expression of p75 may represent a hallmark for EC commitment to death. However, because of the correlative nature of these observations, no definite conclusion could be drawn regarding a possible causative role of NGF system in diabetes-induced microvascular dysfunction. 
For further insight into this matter, we evaluated whether correction of NGF deficits by a supply-side approach could prevent microvascular liabilities and favour reparative angiogenesis. As expected, NGF improved the capillarisation of diabetic wounds. This effect was attributable to stimulation of EC proliferation and inhibition of apoptosis. In addition, NGF supplementation produced significant expressional changes of its own receptors, leading to a 19 -fold increase in TrkA : p75 ratio, thus indirectly confirming the importance of an appropriate balance between these two receptors for a proper healing.

It was somehow surprising that supplementation with NGF did not enhance wound capillarisation in non-diabetic mice. However, evidence indicates that NGF is physiologically up-regulated in healthy animals subjected to tissue injury $[13,14]$. The increase in endogenous NGF could be sufficient to saturate receptor sites, thus making irrelevant the contribution of additional supplements. Furthermore, other reparative mechanisms controlling neoangiogenesis are efficiently activated in non-diabetic animals.

NGF may also act as an indirect activator of EC survival and growth by stimulating the release of other endothelial mitogens including VEGF or modulators of angiogenesis, such as NO. In vivo, we observed that $V E G F-A$ expression is activated in diabetic ulcers treated with NGF. Furthermore, NGF stimulated the release of VEGF-A from cultured ECs and inhibited high-glucose-induced apoptosis, the latter effect being reversed by an antagonist against VEGF-A receptor-2. These findings are consistent with our previous studies documenting the ability of NGF to improve postischaemic neovascularisation of ischaemic hindlimb muscles through a VEGF-A/Akt-B/eNOS-dependent mechanism [14]. The present report adds the important and novel information that VEGF-A is crucial for NGF anti-apoptotic activity.

In conclusion, our results support the hypothesis that a deficit in endogenous NGF production, and upregulation of NGF $\mathrm{p} 75$ receptor may contribute to diabetes-related impairment of reparative angiogenesis. Conversely, topical NGF supplementation accelerates diabetic wound healing by enhancing the regenerative potential of ECs and conferring upon them resistance against apoptotic death, seemingly via modulation of its own receptors and an increase in VEGF-A levels. This study unravels new mechanisms of the healing effect of NGF and provides novel targets for the treatment of diabetic complications.

Acknowledgements. This study was founded by a grant from the Juvenile Diabetes Research Foundation (JDRF, USA no. 1-2001-877) to P. Madeddu. C. D. Figueroa contributed to this study during his stay at the INBB laboratory, and was supported by a grant aimed at favouring investigators coming from developing countries (released from Assessorato Pubblica Istruzione of Sardinia to P. Madeddu).

\section{References}

1. Currie CJ, Morgan CL, Peters JR (1998) Epidemiology and cost of inpatients care of peripheral vascular disease, infection, neuropathy and ulceration in diabetes. Diabetes Care 21:42-48

2. Emanueli C, Salis MB, Pinna A et al. (2002) Prevention of diabetes-induced microangiopathy by human tissue kallikrein gene transfer. Circulation 106:993-997

3. Rivard A, Silver M, Chen D et al. (1999) Rescue of diabetes-related impairment of angiogenesis by intramuscular gene therapy with adeno-VEGF. Am J Pathol 154: 355-363

4. Brown DL, Kane CD, Chernausek SD, Greenhalgh DG (1997) Differential expression of insulin-like growth factors I and II in cutaneous wounds of diabetic and nondiabetic mice. Am J Pathol 151:715-724

5. Ido Y, Carling D, Ruderman N (2002) Hyperglycemiainduced apoptosis in human umbilical vein endothelial cells. Inhibition by the AMP-activated protein kinase activation. Diabetes 51:159-167

6. Frustaci A, Kajstura J, Chimenti C et al. (2000) Myocardial cell death in human diabetes. Circ Res 87:1123-1132

7. Lewin GR, Barde YA (1996) Physiology of the neurotrophins. Ann Rev Neurosci 154:289-317

8. Anand P, Terenghi G, Warner G, Kopelman P, WilliamChestnuts RE, Sinicropi DV (1996) The role of endogenous nerve growth factor in human diabetic neuropathy. Nat Med 2:703-707

9. Unger JW, Klitzsch T, Pera, S, Reiter R (1998) Nerve growth factor (NGF) and diabetic neuropathy in the rat: morphological investigations of the sural nerve, dorsal root ganglion, and spinal cord. Exp Neurol 153:23-24

10. Tuveri M, Generini S, Matucci Cerinic M, Aloe L (2000) NGF, a useful tool in the treatment of chronic vasculitic ulcers in rheumatoid arthritis. Lancet 356:1739-1740

11. Bernabei R, Landi F, Bonini S et al. (1999) Effect of topical application of nerve-growth factor on pressure ulcers. Lancet 354:307

12. Landi F, Aloe L, Russo A, Cesari M et al. (2003) Topical treatment of pressure ulcers with nerve growth factor: a randomized clinical trial. Ann Intern Med 139:635-641

13. Matsuda H, Koyama H, Sato H et al. (1998) Role of nerve growth factor in cutaneous wound healing: accelerating effects in normal and healing-impaired diabetic mice. J Exp Med 187:297-306

14. Emanueli C, Salis MB, Pinna A, Graiani G, Manni L, Madeddu P (2002) Nerve growth factor promotes angiogenesis and arteriogenesis in ischemic hindlimbs. Circulation 106:2257-2262

15. Cantarella G, Lempereur L, Presta M et al. (2002) Nerve growth factor-endothelial cell interaction leads to angiogenesis in vitro and in vivo. FASEB J 16:1037-1039

16. Wang S, Bray P, McCaffrey T, March K, Hempstead BL, Kraemer R (2000) p75(NTR) mediates neurotrophininduced apoptosis of vascular smooth muscle cells. Am J Pathol 157:1247-1258

17. Chao M, Casaccia-Bonnefil P, Carter B, Chittka A, Kong H, Yoon SO (1998) Neurotrophin receptors: mediators of life and death. Brain Res Rev 26:295-301

18. Patapoutian A, Reichardt LF (2001) Trk receptors: mediators of neurotrophin action. Curr Opin Neurobiol 11:272-280

19. Frade JM, Rodriguez-Tebar A, Barde YA (1996) Induction of cell death by endogenous nerve growth factor through its p75 receptor. Nature 383:166-168 
20. Kraemer R (2002) Reduced apoptosis and increased lesion development in the flow-restricted carotid artery of p75(NTR)-null mutant mice. Circ Res 91:494-500

21. Bocchini G, Angeletti PU (1969) The nerve growth factor: Purification as a 30.000-molecular weight protein. Proc Nat Acad Sci USA 64:787-794

22. Di Marco E, Marchisio PC, Bondanza S et al. (1991) Growth regulated synthesis and secretion of biologically active nerve growth factor by human keratinocytes. J Biol Chem 266:21718-217122

23. Derby IA, Bisucci T, Hewitson TD, MacLellan DG (1997) Apoptosis is increased in a model of diabetes-impaired wound healing in genetically diabetic mice. Int $\mathrm{J}$ Biochem Cell Biol 29:191-200

24. Lopez-Sanchez N, Frade JM (2002) Control of the cell cycle by neurotrophins. Lessons from the p75 neurotrophin receptor. Histol Histopatol 17: 1227-1237
25. York RD, Molliver DC, Grewal SS, Stenberg PE, McCleskey EW, Stork PJ (2000) Role of phosphoinositide 3-kinase and endocytosis in nerve growth factor-induced extracellular signal-regulated kinase activation via Ras and Rap1. Mol Cell Biol 20:8069-8083

26. Casademunt E, Carter BD, Benzel I, Frade JM, Dechant G, Barde YA (1999) The zinc finger protein NRIF interacts with the neurotrophin receptor p75(NTR) and participates in programmed cell death. EMBO J 18:6050-6061

27. Salehi AH, Xanthoudakis S, Barker PA (2002) NRAGE, a p75 neurotrophin receptor-interacting protein, induces caspase activation and cell death through a JNK-dependent mitochondrial pathway. J Biol Chem 277:48043-48050

28. Dobrowsky RT, Werner MH, Castellino AM, Chao MV, Hannun YA (1994) Activation of the sphingomyelin cycle through the low-affinity neurotrophin receptor. Science 265:1596-1599 